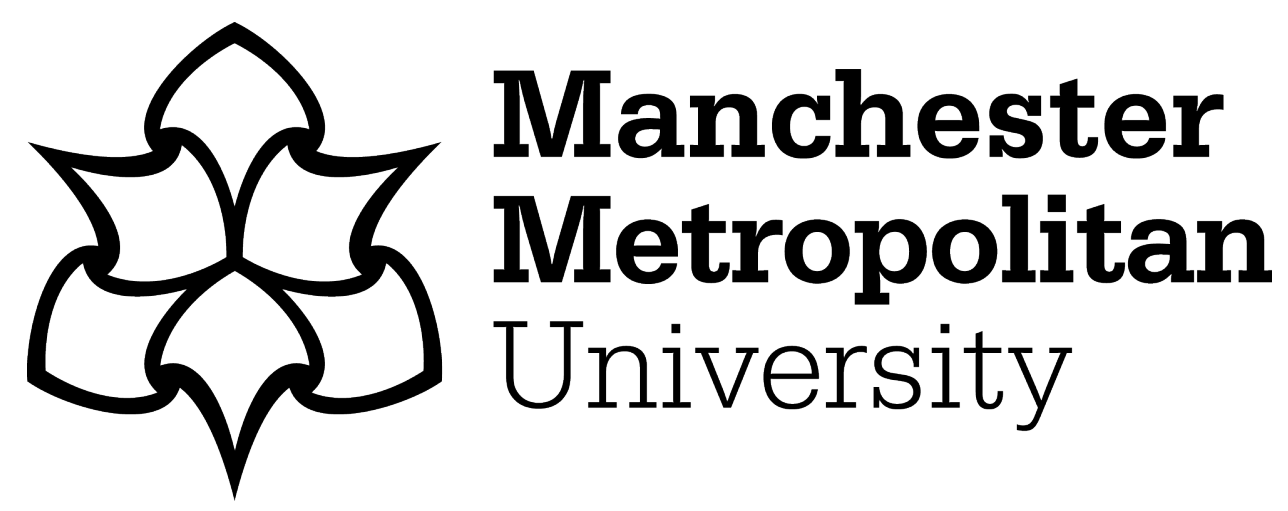

Knight-Percival, Alexander, Johnson, Christopher ORCID logoORCID: https://orcid.org/0000-0003-1172-1383, Richards, Benjamin, Palmer, Scott and Bowring, Nicholas (2020) Mapping of the electromagnetic environment on the railway: condition monitoring of signalling assets. Proceedings of the Institution of Mechanical Engineers, Part F: Journal of Rail and Rapid Transit, 243 (3). pp. 246-256. ISSN 0954-4097

Downloaded from: https://e-space.mmu.ac.uk/620270/

Version: Accepted Version

Publisher: SAGE Publications

DOI: https://doi.org/10.1177/0954409718802998

Please cite the published version 


\title{
Mapping of the electromagnetic environment on the railway: Condition monitoring of signalling assets
}

\author{
Alexander Knight-Percival ${ }^{1,2}$, Christopher Johnson ${ }^{1}$, \\ Benjamin Richards ${ }^{2}$, Scott Palmer ${ }^{2}$ and Nicholas Bowring ${ }^{3}$
}

\begin{abstract}
Conventional track circuit condition monitoring systems are fixed at the wayside, with each installation reporting on a single track circuit. In this work, we present a custom-built, sensitive, magnetic field detection system, which can be fitted to the underside of a rail vehicle. With this system installed, some characteristics of an operating track circuit can be monitored from the vehicle whilst it is in motion. By using appropriate analysis techniques, it is possible to identify the signatures of equipment relating to audio frequency track circuits, the topic of this work. Analysing the signatures of track circuit equipment demonstrated that there were clear differences between track circuit assets. By building on other research into the behaviours of failing track circuits, and continuing to conduct this research, the authors believe that it is possible, and beneficial, to perform condition monitoring of track circuits from low-cost equipment mounted on the train. Coupling this with advanced analysis techniques will allow predictive maintenance of track circuits with very little capital outlay.
\end{abstract}

\section{Keywords}

Railway, electromagnetism, track circuits, audio frequency, Train Protection and Warning System (TPWS), systems engineering, Industry 4.0

\section{Introduction}

While passenger journeys in the United Kingdom decreased to 416 million in Q1 2017-2018 (i.e. by $2.7 \%$ ), the number of passenger kilometres increased to 16.3 billion. ${ }^{1}$ This reflects a trend in passengers using alternative methods of transport for shorter journeys. Rail transport tends to be used for journeys greater than 25 miles. Over half the journeys in England were for commuting, with these being the least satisfied users. Punctuality and reliability of services remain a key driver in passenger satisfaction. ${ }^{2}$ In 2015/2016, Network Rail recorded 7.72 million delay minutes accounting for over $60 \%$ of the delays caused to passengers in England and Wales. ${ }^{3}$

Track circuits are used to protect around half of the United Kingdom's railway network. Failures of track circuit systems account for a significant proportion of delays. In 2012, over 15,500 signalling failures were recorded with delays exceeding 10 min per incident. ${ }^{5}$ On the UK mainline rail, there are approximately 50,991 track circuits in operation. These are of various types and include direct current (DC)- and alternating current (AC)-driven track circuits. Network Rail's asset register describes the specific units on the network. The types of failure and numbers of these units are not readily published; however, Weston et al. ${ }^{4}$ documented track circuits installed within Network Rail's Southern Zone including failure rates. This information is reproduced in Table 1.

Network Rail recorded over 2.6 million delay minutes related to various asset defects including track circuits and axle counters in 2013/2014. ${ }^{6}$ This is a drop from the same incident type in 2008 of $6 \% ; 7^{7}$ however, these numbers have been increasing steadily since, and assuming linear projections are now likely to have reached similar levels. Industry 4.0 has been coined to describe manufacturing systems that operate with a degree of automation and make use of data exchange to facilitate efficient production and systems management. This ideology extends and supports the interdisciplinary field of systems engineering, which

\footnotetext{
${ }^{1}$ Manchester Metropolitan University, Manchester, UK

'Unipart Rail, Doncaster, UK

${ }^{3}$ University of Huddersfield, Huddersfield, UK

Corresponding author:

Alexander Knight-Percival, Research and Innovation, Unipart Rail,

Jupiter Building, First Point, Doncaster, DN4 5JQ, UK.

Email: alex.knight-percival@unipartrail.com
} 
Table I. Typical track circuit failure statistics. ${ }^{4}$

\begin{tabular}{lcclr}
\hline $\begin{array}{l}\text { Track circuit } \\
\text { type }\end{array}$ & Number & $\begin{array}{l}\text { Failure } \\
\text { reports } \\
\text { per year }\end{array}$ & $\begin{array}{l}\text { Failure rate } \\
\text { per year }\end{array}$ & $\begin{array}{l}\text { Impact } \\
\text { minutes }\end{array}$ \\
\hline AC-TC & 3643 & 1264 & 0.347 & 158,000 \\
TI2I & 1326 & 524 & 0.395 & 65,500 \\
FS2600 & 528 & $24 I$ & 0.456 & 30,125 \\
HVI TC & 952 & 390 & 0.410 & 48,750 \\
Reed TC & 1895 & 1304 & 0.688 & 163,000 \\
Overall & 8344 & 3723 & 0.446 & 465,375 \\
\hline
\end{tabular}

focuses on the design and management of complex systems over their life cycles. To facilitate this, Industry 4.0 works with four design principles. Interoperability, the ability of systems, devices and sensors to connect and communicate with each other using automated processes. Information transparency, using a virtual digital twin of the physical system integrating sensor data and allowing contextualisation of the systems state. Technical assistance, allowing a systems engineering holistic approach to manage a complex system by visualising complex data and so reducing workload. Decentralized decisions, allowing a range of autonomy so that complex systems can adapt to their environment within given tolerances.

Condition monitoring of track circuit systems can help to solve availability issues by analysing a working track circuit and looking for early indications of failures, allowing preventative maintenance to be carried out before the system stops operating. Various studies have looked into condition monitoring of track circuits. To provide a fully aligned Industry 4.0 view of a track circuit, both a digital twin and a level of intelligence within the condition monitoring equipment must be applied. The following article looks into the application of a standoff locomotive-based monitoring equipment that could be supported by existing digital twin models discussed within the literature review which could integrate measurements of track circuit behaviours.

Conventional track circuit condition monitoring systems are fixed at the wayside, with each installation reporting on a single track circuit. The use of standoff locomotive-based monitoring equipment removes the need to instrument or for the renewal of wayside equipment. This reduces installation cost and maintenance cost as additional complexity is avoided. Additional benefits include the ability to process data centrally allowing future innovation to be evaluated through means of benchmark testing before the application of any decentralised decision process.

\section{Background}

Condition monitoring of track circuits has been well documented in past publications. Weston et al. discussed the application of condition monitoring on TI21 track circuits in the UK. ${ }^{4}$ Through evaluating the transmission and receiver characteristics of track circuits that had been stable, some useful data were identified. Key findings included the relationship of received and transmitted current to weather conditions. While temperature variations were identifiable, this had little effect on the transmitted signal. Significant attenuation was observed in wet conditions with rain causing a drop of $5 \%$ in the transmitted current, resulting in $19 \%$ of attenuation in the received signal. It was noted that the quantity of rain had little effect.

It was postulated that the reduction in shunt resistance caused by rain and the reduced ballast and sleeper resistance could be causing a reduction in the Q-factor of the tuned circuit. A similar effect could be possible on the received end.

Bell presented an application of a track circuit data logger for DC- and AC-type track circuits. ${ }^{8}$ The effects of the ballast conditions are highlighted as causing issues with track circuit resilience. However, Bell also discussed preliminary complications with track circuits and railhead contamination. In the UK, this is usually due to leaf fall, and can cause a more significant wrong side signal failure, where a train's presence is not detected. An equivalent circuit of the track and track relay for modelling is presented, with discussion around the effect of noise from locomotive traction systems. The effect of this noise in partially energising the relay coil has minimal effect as the track relay is relatively immune to the effect of noise.

Current monitoring data gathered from a DC track circuit are presented showing the effect of the overhead line equipment. While significant noise can be observed, this is easily filtered. Similar noise has been observed during track circuit measurements in line-side environments, further indicating that electromagnetic issues are minimal.

Saade et al. builds upon the work of Weston et al. in evaluating both environmental effects and those of track circuit assistors. ${ }^{9}$ A condition monitoring piece of equipment is described in more detail, with the development of some automated analysis in evaluating the audio frequency components through Fourier analysis. The diurnal effect of temperature is observed again as with Weston et al. with the warmest part of the day showing lowest root mean square (RMS) voltage on the receiver end.

The temperature impact on audio frequency was evaluated in more detail by Zanwu Huang et al. by applying linear regression. ${ }^{10}$ In this analysis, only measurements where rainfall is zero are used. The voltage and current measurements of the transmitter and receiver are reviewed in detail. While transmission voltage is observed to be affected by temperature, the receiver tuning unit current is observed to be 
unaffected. This further enforces that temperature drift plays little effect in track circuit failure.

A comprehensive review of condition monitoring of 385 audio frequency track circuits on London Underground is presented by Etcell et al. ${ }^{11}$ The application of the infrastructure required to monitor these track circuits is described in detail. A standardised architecture was constructed using commercial off-theshelf equipment, involving the use of high-end field programmable gate arrays to allow processing and transmission of the acquired data in real-time. The integration of a graphical user interface in order to visualise these data brings the application into the realms of Industry 4.0. By centrally processing the acquired data, their system intelligence is able to proactively predict failures of equipment before a critical failure occurs.

The application of pattern recognition and artificial intelligence in order to classify the track circuit failure types has been shown to be effective. Such methods would provide the foundation for a decentralised decision-making process and allow predictive maintenance to be delivered effectively. Chen et al. investigated the ability of neuro-fuzzy systems to detect and classify failures of audio frequency track circuits. ${ }^{12}$ Within a laboratory environment, 11 operating modes are evaluated with operating characteristics sampled for ideal operation and 11 fault conditions. A co-active neuro-fuzzy inference system (CANFIS) model is applied, due to its capability in solving poorly defined problems. Artificial noise is introduced within the test set to evaluate the robustness of the CANFIS model. With $10 \%$ noise, correct classification of $98.27 \%$ is realised with this falling below $90 \%$ when noise is increased to $30 \%$. As seen in the work by Bell, the level of noise can be reduced through sufficient filtering. ${ }^{8}$

Bruin et al. demonstrate similar results using recurrent neural networks. ${ }^{13}$ The stochastic nature of this neural network type provides additional resilience by evaluating previous decision data within its current decision cycle. This allows the consideration of time-based analysis within its evaluation of the operating state of the track circuits. Only five fault types are considered in this application, but importantly ballast degradation is included within these failure types. Again, a high success rate of $99.7 \%$ is observed; however, it is worth noting that data are obtained through a generative model of track circuit behaviour based on a smaller sampling of actual track circuit behaviour.

Interesting notes on the failure conditions of the recurrent neural network show that knowledge of misclassifications is required in order to identify the true likely cause of wrongly classified failures. Similar experience is essential in current operations in the rail network.
Zhao and Shi present a comprehensive investigation into track circuit readers used on Chinese railways. ${ }^{14}$ The Chinese Train Control Systems (CTCS) has several levels similar to the developing European Train Control System. In their paper, Zhao and Shi focus on audio frequency jointless track circuits. Within CTCS, the train speed for each track section is modulated with the track circuit signal. The track circuit reader consists of a pair of trainborne antennae that allow the induced magnetic field to be observed and the speed signal demodulated for train control.

A model of the electromagnetic field is compared to lab-based experiments to allow accurate modelling using transmission line theory and to evaluate working range in relationship to vehicle speed. Their findings indicate that track circuit readers have an effective range of $3 \mathrm{~m}$ and the largest induced magnetic flux is at $1.5 \mathrm{~m}$, i.e. halfway between the shunt point and the signal origin, this being the mounting point of track circuit reader.

A key finding from Zhao and Shi is that there is a linear relationship between the jointless track circuit current and the voltage induced in the track circuit reader and that the phase difference is not affected by the velocity of the train. Therefore, by using appropriate analysis techniques and a trainborne sensor, signatures of equipment relating to audio frequency track circuits can be identified for the purpose of condition monitoring, the topic of this work.

\section{Track circuit operating principles}

A track circuit system uses an electrical signal to detect the occupancy of a section of track. It relies on electrical conductivity along the running rails and across the wheelset of the locomotive to operate correctly. The electrical signal may be DC or AC.

Where the track is protected by track circuits, the railway is divided into discrete blocks which are electrically isolated from one another. A DC track circuit achieves isolation by physically separating sections of track using insulated rail joints. Some AC track circuit systems use an electrical device called a tuning unit in place of insulated joints. These devices connect to the running rails and are tuned to a track circuit's specific frequency. They heavily attenuate the track circuit signal over a short distance, effectively emulating the effect of an insulated joint. This allows these types of AC track circuits to operate on continuously welded rails.

The flow of current in a simplified track circuit system is presented in Figure 1. The track circuit system consists of a transmitter which conducts an electrical signal along the rails to a receiver. If the receiver detects the signal with sufficient amplitude, the section of track is presumed to be empty. But when a 


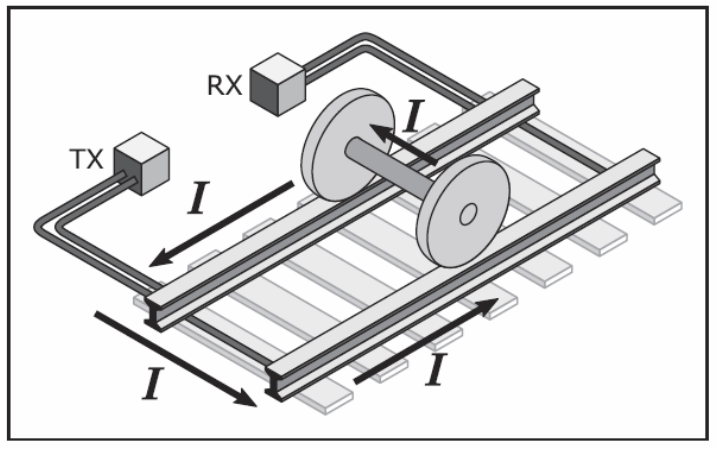

Figure 1. A simplified model of a track circuit during shunting, showing how current flows from the track circuit transmitter (TX), around the rails and through the leading wheelset of the locomotive. The current reaching the receiver $(\mathrm{RX})$ is small during shunting, as the majority of the current is conducted across the wheelset, which is the path of lowest electrical resistance.

train is present, as illustrated, there exists a high conductivity path across the running rails, which reduces the signal reaching the receiver. If the signal level falls below a defined threshold, the section of track is occupied.

Faults that can affect a track circuit system include failures of the transmitters or receivers, cable connections, tuning units and track termination bonds. ${ }^{15}$ Factors not directly related to the track circuit equipment, including rail conductance impairments and ballast degradation, can also cause the system to fail. ${ }^{13}$ A large increase in the shunt resistance of the train can also have an effect, ${ }^{16}$ although this can be mitigated through the use of additional equipment such as a track circuit assistor. ${ }^{17}$

For this investigation, analysis is limited to the EBI Track 200 (also referred to as TI21)-type track circuits, as these were the type of track circuit prevalent in the test areas. These track circuits use an AC signal and tend to use tuning units to separate adjacent sections of track. This work assumes a negligible shunting resistance, and the effect of a finite resistance has been considered in other work. ${ }^{18}$

\section{Data collection}

Measuring track circuits using a trainborne, noncontact method may be carried out using the principle of electromagnetic induction.

The current flowing through each track circuit generates a magnetic field, detectable in the region around the rails. The field strength is proportional to the current flowing around the track circuit. The direction and strength of the magnetic field, normally expressed as the vector quantity $\mathrm{B}$, depends mainly on the position at which the field is measured, and the specific geometry of the current loop formed by the track circuit. Other factors to consider are the effects of the surrounding materials, including the metalwork of the locomotive, and the effect of material around the rails, including the ballast.

\section{Equipment}

For an AC track circuit, like the EBI 200, the magnitude of the magnetic field oscillates with the electric current in the track circuit. This changing magnetic field will induce current in a conductor. To detect this, a sensing element was constructed from of a coil of conductive wire wound around a ferrite rod. The wire is sensitive to time-varying magnetic fields, such as those produced by an AC track circuit. The coil geometry reacts to the changing $\mathrm{B}$ field along an axis perpendicular to the coil windings, with each winding amplifying the sensitivity of the detector. The ferrite acts to improve the sensitivity of the detector further, by concentrating the field in the vicinity of the coil.

The sensing element is designed to allow signals to be captured efficiently over a wide band. However, as the sensing element is responsive only to a changing magnetic field, the sensitivity necessarily drops off towards lower frequencies. The electrical properties of the coil cause a resonance which amplifies the response around $120 \mathrm{kHz}$. The impedance of the sensor also increases at higher frequencies, damping the response.

To protect the sensing element, it is potted inside Unipart Rail's standard low-profile TPWS aerial housing. The housing, which is already rail-certified, provides the mechanical strength for the sensor to withstand the harsh vibrational environment on the bogie. A specialised mounting plate was built to mount the sensor housing under the vehicle and next to the TPWS aerial, which is located at the vehicle's leading wheelset. This location is near to the current loop formed by the track circuit, assuming that the transmitter is ahead of the train, allowing the magnetic field from the track circuit to be measured effectively.

The remainder of the equipment used to obtain the data is shown in Figure 2. In addition to the sensing element described above, a global positioning system (GPS) receiver captures the geographic coordinates of the locomotive, and digital recording equipment stores all of the captured data for later analysis.

The digital recording equipment measures the potential difference over the sensing element and converts this to a digital value, using an analogue-todigital converter (ADC) with a resolution of eight bits. The ADC was set to measure the aerial voltage every $640 \mathrm{~ns}$, giving a frequency resolution of $800 \mathrm{kHz}$. These measurements are continuously recorded to the storage device. The recording equipment is located in the cab of the locomotive, away from the sensing element, where it is subject to far less vibration and can be accessed by the research team. A length of shielded 
cable temporarily fixed to the body of the train connects the recording equipment to the sensing element. The

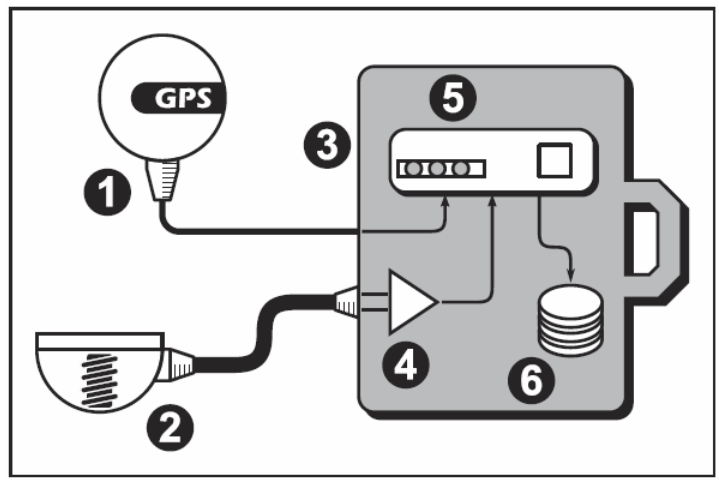

Figure 2. The detection kit, consisting of (1) GPS receiver, (2) sensing element, (3) carrying case for digital recording equipment, (4) ADC, (5) miniature computer, and (6) storage medium.

high sample rate ensures that faster transient signals can be captured, as well as higher order harmonics of the track circuit signal. For later work based on this research, it may be possible to select a lower sample rate, depending on the signals of interest.

The GPS receiver is fitted in the cab, in the window where it has a good view of the sky. It continually computes the geographic coordinate of the locomotive, which is recorded to the storage device along with the sensing element data. These location data are used later to determine the alignment of the aerial voltage measurements along the test route.

During the recording process, a specialised program running on the measurement computer is used to stream ADC and GPS data to the storage medium. Data from the ADC device are transmitted to the computer in variable length frames containing up to several thousand samples each. The GPS receiver transmits updates approximately once per second, using the standard National Marine Electronics Association 0183 (NMEA) format. The size of the entire NMEA message is negligible compared to the ADC data frames, and so every message is saved to the storage medium in its entirety.

The data are encoded using a simplistic file format, which is designed to maximise throughput and minimise random access. Both GPS timestamps and those from the computer's on-board clock are recorded periodically. This ensures that samples are correctly time-indexed even before a GPS fix is obtained, and so can be aligned with other samples that have a GPS location recorded. Additionally, any missing frames that may occur do not cause the data to be misaligned.

\section{Data capture}

The equipment described in the previous section was fitted to an in-service locomotive. Figure 3 is a

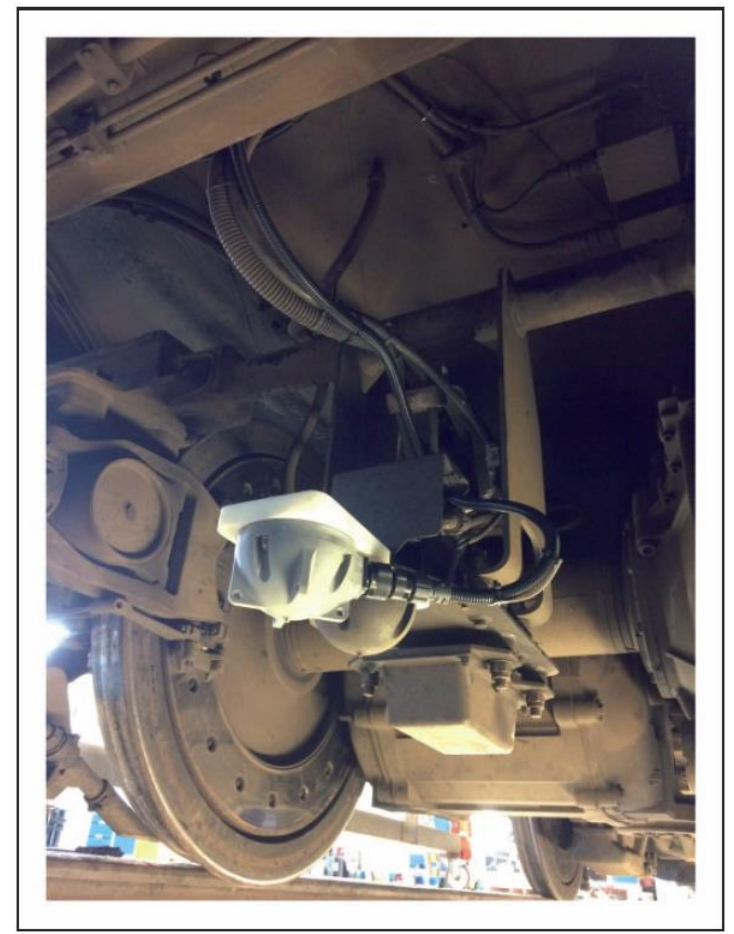

Figure 3. The sensing element, potted inside a Unipart Rail TPWS housing and fitted to the underside of a locomotive, at the leading axle.

photograph of the sensor mounted on the underside of the bogie.

Figure 3. The sensing element, potted inside a Unipart Rail TPWS housing and fitted to the underside of a locomotive, at the leading axle.

During the trial runs, the locomotive was driven over several hundred miles of the UK mainline railway network. Much of the distance covered was at full line speed, which was at most $90 \mathrm{mph}$ in this case. There were also periods when moving at a reduced speed, due to a yellow signal, or when stopped completely, at red signals or at stations.

Overall, this was representative of the different types of driving that could affect the measurement equipment. However, there was not any significant effect on the analysis described in the next section.

Approximately half of the distance covered was found to be protected by EBI 200-type track circuits.

\section{Analysis}

After data collection was complete, the storage device can be removed, and the dataset copied to another computer for analysis. Due to the large volume of data collected, it is not instructive to interpret the data directly in the measurement space. Analysis of the data was performed in two parts. First, the data were transformed into a reduced feature space, to discard information in the signal not relevant to EBI 200 track circuits. Following this, the GPS information recorded during the trial was used to map the feature space onto 
geographic coordinates. This allowed the location of the track circuit features to be correlated to the route of the train.

The location accuracy of the position of the track circuit features was limited by the GPS accuracy, and a systematic error introduced by the delay in receiving NMEA data from the GPS receiver. At the highest line speed of $90 \mathrm{mph}$, the combination of these error sources would give a worst-case positional accuracy of $40 \mathrm{~m}$. This improves as the speed is reduced. For the purposes of this work, the positional inaccuracy was neglected, as the data are not currently compared to historical data. An improved system would use a higher grade GPS receiver, combined with sensor fusion techniques to improve location accuracy to below a centimetre. ${ }^{19}$

\section{Feature extraction}

The sampling rate of the ADC was such that $1.6 \mathrm{M}$ samples were recorded per second. To identify EBI 200 track circuits within the recorded signal, it is convenient to make use of their characteristic frequency, which could take one of their eight nominal frequency values (referred to by the letters $\mathrm{A}-\mathrm{H}$ ). ${ }^{20}$ The operating frequencies for EBI 200 track circuits are given in Table 2. Each EBI 200 track circuit operates using the frequency pair listed in the third column. The transmitter alternates between transmitting on the two carrier frequencies at a characteristic modulation frequency of $4.8 \mathrm{~Hz}$.

\begin{tabular}{lll}
\hline Frequency & Nominal $(\mathrm{Hz})$ & Actual $(\mathrm{Hz})$ \\
\hline A & 1699 & 1682,1716 \\
B & 2296 & 2279,2313 \\
C & 1996 & 1979,2013 \\
D & 2593 & 2576,2610 \\
E & 1549 & 1532,1566 \\
F & 2146 & 2129,2163 \\
G & 1848 & 1831,1865 \\
H & 2445 & 2428,2462 \\
\hline
\end{tabular}

A short-time Fourier transform (STFT) method $^{21}$ was used to transform the time-domain measurement data $x(n)$ into the frequency domain. The frame size $M$ was chosen to be $2^{17}=131072$ samples, which corresponds to $84 \mathrm{~ms}$ of data. A power of two was used here as this increases computational efficiency when performing a fast Fourier transform. A discrete time Fourier transform (DTFT) was applied to each data frame by applying a window function $w(n)$ to the data.

$$
X_{m}(\omega)=\sum_{n=0}^{N-1} x(n) w(n-m R) e^{-i \omega n}
$$

A hop size of $R$ was chosen as half the frame size to allow overlap between the successive transforms. The window function is defined to remove data outside of the frame $m$, and to apply a Hann window, to smooth discontinuities at the beginning and end of the frame.

$$
w(n)=\frac{1}{2}\left(1-\cos \left(\frac{2 \pi x(n)}{M-1}\right)\right) \quad 0 \leqslant n \leqslant M-1
$$

In the frequency domain, the frame size gives a frequency resolution of $12 \mathrm{~Hz}$, which is sufficient to resolve the track circuit frequencies from one another, and also to begin to detect the $\pm 17 \mathrm{~Hz}$ modulation at each frequency.

The result of transforming into the frequency space is shown in Figure 4, which has been calculated using a larger frame size compared to the main analysis, in order to show the detail in the waveform. A succession of DTFTs, for successive frames, are visualised as a stack of plots spanning just under one second. For each complex value at each DTFT frequency bin, the power was calculated from the magnitude squared as $|X m(\omega)|^{2}$ to produce the vertical axis. In this example, the track circuit alternates between $2567 \mathrm{~Hz}$ and $2610 \mathrm{~Hz}$ with a

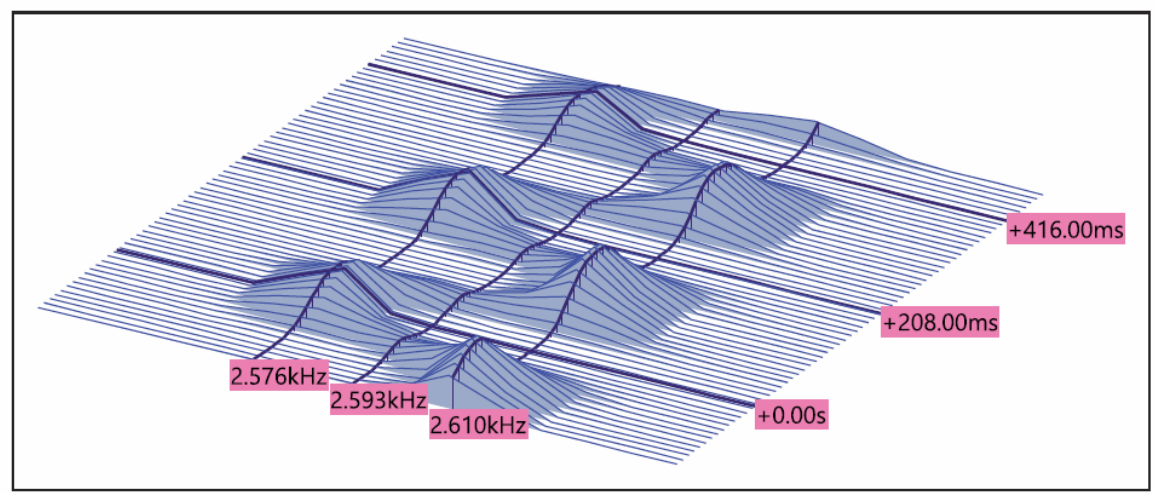

Figure 4. A spectrogram, showing the power of a succession of STFT of detected EBI 200 modulation $\pm 17 \mathrm{~Hz}$ around frequency $\mathrm{D}$, nominally $2593 \mathrm{~Hz}$. The time axis is shown going into the page, and the frequency axis increases towards the right of the figure. The detected and nominal frequencies are marked, as are the time periods corresponding to the $4.8 \mathrm{~Hz}$ modulation. The remaining dimension, visualised as the height of the peaks, represents the amplitude of the detected signal, given in arbitrary units. 
frequency of $4.8 \mathrm{~Hz}$. This corresponds to the detection of a frequency D EBI 200 track circuit.

To transform the frequency data into a feature space, each STFT segment was assigned a score, calculated from the frequency content detected in the track circuit operating range. To generate a score for each data frame, each DTFT segment was multiplied by a weighting function that was nonzero around the track circuit frequency range of interest.

For each of the EBI 200 track circuit frequencies, a weighting function was defined as a simple 'top hat' with a bandwidth $\frac{B}{2 \pi}=B_{\mathrm{Hz}}$ of $100 \mathrm{~Hz}$, centred around each nominal transmitter frequency. This gave eight different functions which would individually identify their respective transmitter frequencies, $v_{\{A-H\}} \mathrm{Hz}$.

$$
Y_{v}(\omega)= \begin{cases}1, & \text { for }-B / 2 \leqslant \omega-2 \pi v \leqslant+B / 2 \\ 0, & \text { otherwise }\end{cases}
$$

A further function was defined to be nonzero over all eight of the bands covering the track circuit frequencies. This allowed areas where any EBI 200 equipment was present to be identified from the data.

An array of values derived from multiplying DTFT power values with the weighting function were summed, producing a score for each frame.

$$
S(m)=\sum_{\omega}\left|X_{m}(\omega)\right|^{2} Y(w)
$$

This effectively reduced the volume of data by assigning a value to each of the STFT frames, giving a measure related to the amplitude of the track circuit signal detected in the frame for the frequencies of interest.

It is noted that this simplistic algorithm could be improved, for example by locking on to the characteristic modulation of the EBI 200 track circuit signal. However, the method described here proved effective in locating the track circuit signal within the data and so was sufficient to demonstrate the feasibility of the measurement technique.

\section{Geospatial mapping}

Having reduced the data to a smaller feature space, it is then useful to project that dataset onto a twodimensional geographic map of the route.

Freely available maps from the OpenSteetMap ${ }^{22}$ project were used for this purpose. OpenStreetMap allows portions of their map to be downloaded as small images called tiles, rendered at different zoom levels. Each map tile is a representation of the geographic data contained within a defined square area of the map.

Using the GPS data recorded during the trial, a line can be plotted on top of a grid of map tiles, showing the route of the train. Then, an estimate for the position of each data frame was interpolated from the geographic coordinates recorded periodically in the data file.

To display these data in an intuitive manner, the line is coloured depending on the relative score at each position along the route. An example graphic displaying the data is shown in Figure 5. Along the route, the location of the track circuit transmitters is visible in red. Typically, the track circuit transmitters

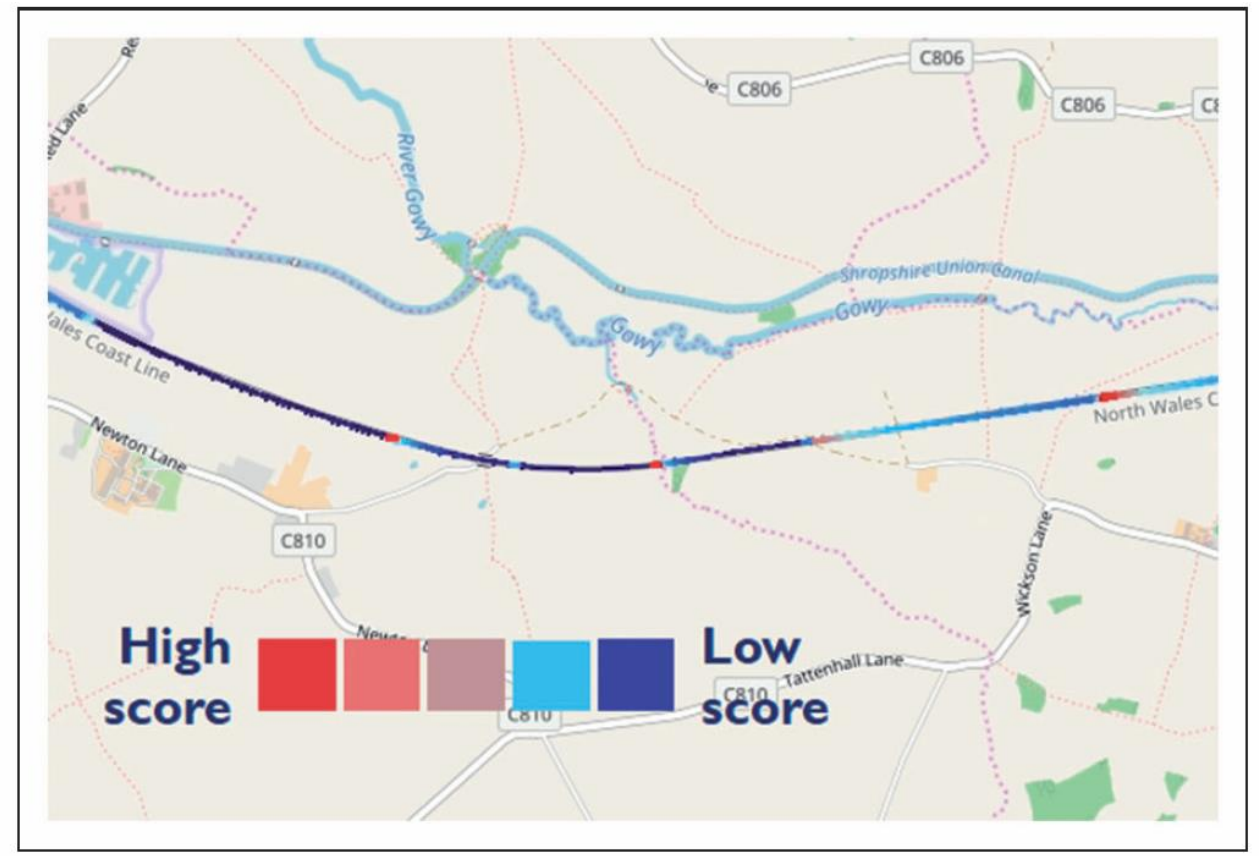

Figure 5. A geographic representation of the data, viewed with the route of the locomotive plotted over an OpenStreetMap geographic map. The line representing the route is coloured to show the relative scoring of each STFT frame. The train is heading north-west. 
were found around $1 \mathrm{~km}$ apart from one another, which agrees with the documented maximum distance. As the train approaches the transmitter, the physical length of the electrical circuit decreases, as there is less total length of rail between the transmitter and the wheelset. This reduces the resistance and increases the current through the track circuit. The inductive sensor reacts to this with an increased response, and so the map shows a characteristic increase in detected amplitude as the train moves towards each transmitter.

This allows Industry 4.0 alignment with a virtual digital twin of the rail network integrating the sensor system state, allowing a holistic approach when visualising the complex frequency and scoring data.

\section{Condition monitoring}

Condition monitoring can be achieved from these data through several means. The score calculated for each frame is a result of the track circuit current during shunting. The ability to monitor this current at all locations along the route presents opportunities to detect the onset of the failure modes mentioned previously.

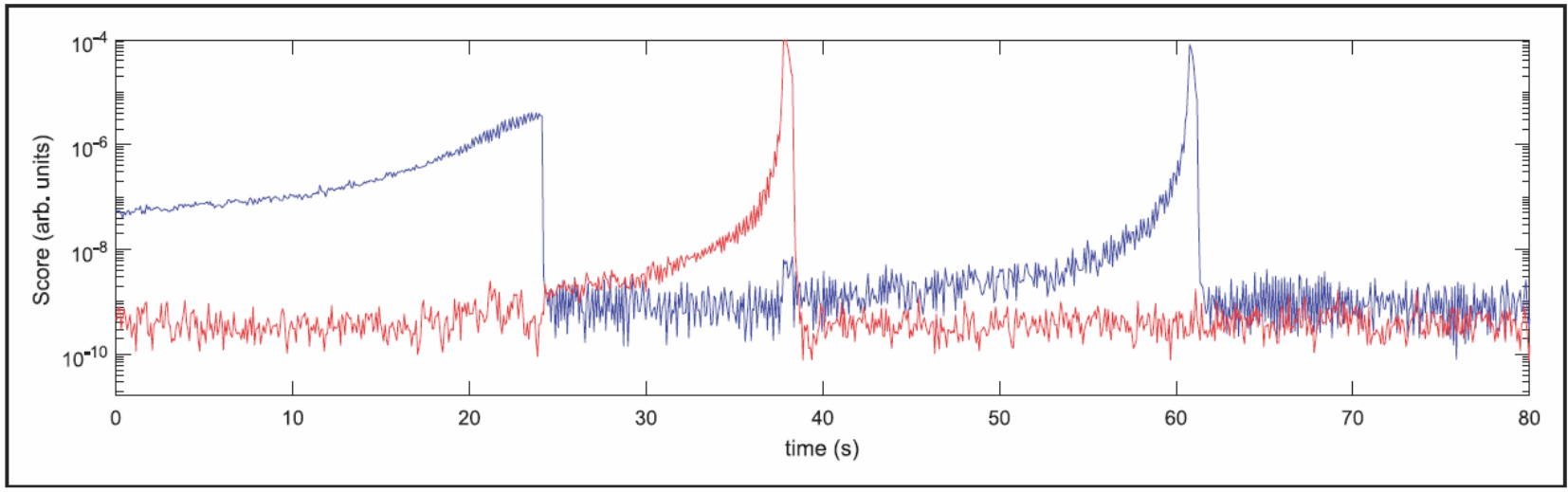

Figure 6. The scoring of the route shown in Figure 5. The scoring is shown calculated separately for the two track circuit frequencies used along this line. The vertical scoring axis is shown with a logarithmic scale.

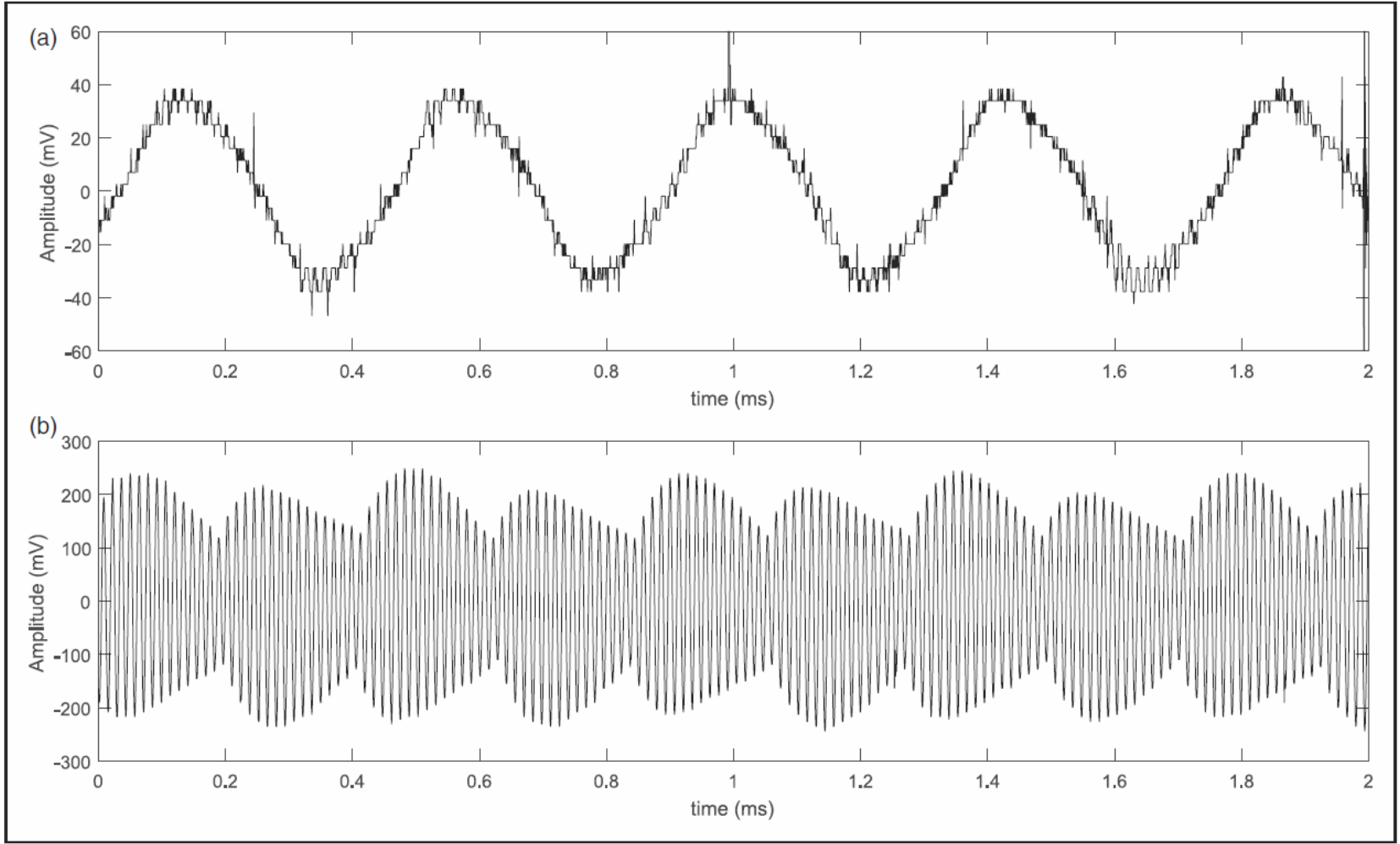

data. This further allows the contextualisation of the

Figure 7. A comparison of two different time-domain waveforms, captured from the sensing element whilst passing over two different EBI 200 track circuit transmitters. In (a), the waveform is smooth, but in (b) the sensor has detected other frequencies in the signal. 
A plot of the scoring profile along the part of the route shown in Figure 5 is given in Figure 6, split into scores for EBI 200 frequencies C and D. Each track circuit transmitter exhibits a distinctive profile leading up to its location. The shape of the profile at each frequency is dependent on the configuration of the track circuits, which may be centre-fed for some sections, and may contain end termination units. ${ }^{20}$ It will also depend on the conductivity profile of the rail and ballast, and the direction that the train travels over the infrastructure. Changes in this profile could indicate the onset of some types of failure. For example, a step change in the conductivity present at one point along the track would manifest itself as a discontinuity in the score profile. By collecting more sensor data over a period of time, it would be possible for a condition monitoring system to detect deviations from the normal profile, and so warn of an impending failure. This type of technique would be useful for detecting failures in the ballast or rail, for example.

Although most of the analysis has concentrated on scoring the track circuit signal in the frequency domain, it is also instructive to examine the time domain signal at some points of interest.

Viewing the data around the areas where track circuit transmitters are located can yield information about the shunting behaviour, and of the track circuit waveform itself.

In Figure 7, a comparison of two different signals at the track circuit transmitter is presented. In Figure 7(a), a signal is presented from a track circuit with very little distortion. Although many track circuits along the route would present some level of distortion, the transmitter signal detected in Figure 7(b) was a clear outlier.

Regions like this are easy to identify in the data, as the signal contains readily detectable higher order harmonic content. When viewed again in the frequency domain, these harmonics are specifically oddnumbered multiples of the track circuit frequency, as with the Fourier components of a square wave. As noted earlier, the inductive sensor used in this investigation does not have a flat response across all frequencies, so the amplitudes of the components do not follow the expected pattern. However, the detection of components at these frequencies may therefore suggest that the EBI 200 waveform is distorted or clipped. The ability to detect such distortions may give an early warning of transmitter failure.

\section{Conclusions}

In this project, the authors experimentally demonstrated the feasibility of measuring properties of track circuits from a moving rail vehicle. This information could then be used to infer the condition of track circuits and make predictions about future failures.

A low-cost sensing element was demonstrated which, along with some commodity hardware, can be used to measure properties of AC track circuits that are due to the current flowing through them. The sensing technique was based on the principle of electromagnetic induction, and the measurement was therefore non-intrusive and galvanically isolated from the track circuit.

With the sensor and associated hardware attached to an in-service locomotive, electromagnetic measurements can be obtained from the track infrastructure. Data were collected over portions of the UK railway network protected by EBI 200 track circuits.

Carrying out analysis in the frequency domain is convenient for rejecting components of the signal away from the track circuit frequencies. The frequency spectrum was computed for each trial dataset over consecutive fixed-sized frames using the DTFT. Each frame was filtered by multiplying the magnitude squared of each of the resulting DTFT points by a weighting function, specified to produce a nonzero result within the EBI 200 track circuit frequency band. The resulting elements were then summed to obtain a score for each of the data frames.

A simple example of condition monitoring was presented, where a specific track circuit transmitter was identified to be emitting a waveform that was shown to have some distortion.

Profiles of the feature space leading up to track circuit transmitters were also obtained. This would allow for further condition predictions to be derived from the data once a series of historical measurements could be taken.

\section{Future work}

In order to continue the work, the authors propose programming a computer model to carry out an electromagnetic simulation of the field produced by the track circuit. This would have several benefits.

The orientation of the sensing element used in the trial, whilst adequate for these measurements, was not necessarily optimal. By modelling the field, it would be possible to determine the most optimal orientation for the sensor. This could then be verified experimentally by installing a three-axis inductive sensor during a later trial.

A theoretical model would also allow verification of the experimentally measured feature space. From here, prediction of the feature space under the onset of various fault conditions could be carried out. The effect of ballast degradation, rail defects and flooding would be of particular interest. 
To enable this, future work will concentrate on automatic classification of condition. Machine learning techniques show some promise in this area. ${ }^{13}$

Further work on the tools to classify the state of the track circuit transmitter will also be carried out. The scoring system presented here is best suited to measuring changes in the amplitude of the track circuit signal. An alternative scoring system designed with an appropriate weighting function and longer STFT window would be able to identify changes in frequency with high resolution, which would also indicate the onset of a failure.

Identifying issues with the track circuit receiver is also a possibility that has not been fully explored in this work. One approach to this may be to measure the current in the tuned zone, to give an indication of the current that is drawn by the device. Such a measurement is technically challenging as the shunting will affect the measurement, and the short length of the tuned zone requires that the measurement is taken quickly.

Furthermore, there still remains risk of significant variation around tuned zones when acquiring data at high speed. The score will depend on how the weighting function falls across the tuned zone, and it is likely that a completed system would discard some condition information from these regions. An alternative method of analysis would be needed for finding such problems.

Areas in which track circuit assisters are used and track circuit actuator interference devices require further investigation as current is potentially diverted through a new path. Although it is noted that the investigated method should be able to inform if the track circuit itself is at fault or rail conductance impairments such as rust are the primary cause of signal failures. Difference may be characterised by the signal attenuation and to a further extent signal attenuation over time.

Finally, a permanent installation of the sensor on a vehicle would then allow further experimental verification and would enable thorough testing of the theoretical modelling and automatic classification tools.

\section{References}

1. Office of Rail and Road. 2017-18 Q1 Statistical release passenger rail usage, http://orr.gov.uk/_data/assets/pdf_file/0006/25719/pass enger-rail-usage-2017-18-q1.pdf (2017). Google Scholar

2. Department for Transport. Rail factsheet, https://assets.publishing.service.gov.uk/government/uplo ads/system/uploads/attachment_data/file/663116/railfactsheet-2017.pdf (2017). Google Scholar

3. Network Rail. Network rail infrastructure limited annual return 2016, https://cdn.networkrail.co.uk/wp-
content/uploads/2016/11/Network-Rail-InfrastructureLimited_Annual-Return-2016.pdf (2016). Google Scholar

4. Weston PF, Chen J, Stewart E, et al. Condition monitoring of audio frequency track circuits. In: 2008 International conference on railway engineering challenges for railway transportation in information age, 2008, pp.1-6. IET. Retrieved from https://ieeexplore.ieee.org/document/4730847/. Google Scholar

5. Network Rail . Number of signalling failures by operating route, London: Office of Rail and Road Statistics, 2012. Google Scholar

6. Network Rail. Network Rail Infrastructure Limited Annual Return 2014, https://cdn.networkrail.co.uk/wpcontent/uploads/2016/11/annual-return-2014.pdf (2014). Google Scholar

7. Network Rail. Network Rail Infrastructure Limited Annual Return 2008, https://cdn.networkrail.co.uk/wpcontent/uploads/2016/11/2008-annual-return.pdf (2008). Google Scholar

8. Bell CR. Remote condition monitoring of phase sensitive AC track circuits. In: 5th IET conference on railway condition monitoring and non-destructive testing (RCM 2011), 2011, pp.1-7. IET. Retrieved from https://ieeexplore.ieee.org/document/6191870/. Google Scholar

9. Saade L, Weston P and Roberts C. Deep monitoring of audio frequency track circuits. In: 6th IET conference on railway condition monitoring (RCM 2014), 2014, pp.15. IET. Retrieved from https://ieeexplore.ieee.org/document/7105023/. Google Scholar

10. Huang Z, Li S and Wei X. Analysis of temperature impact on audio frequency track circuits using linear regression model. In: AIP conference proceedings, vol. 1834 of 1, American Institute of Physics, http://aip.scitation.org/doi/abs/10.1063/1.4981558 (accessed 15 September 2018). Google Scholar

11. Etchell S, Phillips D and Blackburn C. Remote condition monitoring of London underground track circuits utilising commercial off-the-shelf hardware and software platforms. In: 6th IET conference on railway condition monitoring (RCM 2014), 2014, pp.1-5. IET. Retrieved from https://ieeexplore.ieee.org/document/7105014/. Google Scholar

12. Chen, J, Roberts, C, Weston, P. Fault detection and diagnosis for railway track circuits using neuro-fuzzy systems. Control Eng Pract 2008; 16: 585-596. Google Scholar, ISI

13. de Bruin, T, Verbert, K, Babuka, R. Railway track circuit fault diagnosis using recurrent neural networks. IEEE Trans Neural Netw Learn Syst 2017; 28: 523-533. Google Scholar, Medline

14. Zhao, LH, Shi, WS. Induction coupling between jointless track circuits and track-circuit-reader antenna. Prog Electromagn Res 2013; 138: 173-196. Google Scholar

15. Chen, J, Roberts, C, Weston, P. Fault detection and diagnosis for railway track circuits using neuro-fuzzy systems. Control Eng Pract 2008; 16: 585-596. Google Scholar, ISI 
16. Takahashi $\mathrm{S}$ and Nakamura H. Onboard measurement technology on probe train for signaling equipment. In: 2006 SICE-ICASE international joint conference, 2006, pp.1986-1990. Institute of Electrical and Electronics Engineers (IEEE). Retrieved from https://ieeexplore.ieee.org/document/4109012/. Google Scholar

17. Unipart Rail. Track Circuit Assistor, 2018. Brochure, www.unipartrail.com/assets/tca-2pp-aus-brochurest1.pdf (accessed 15 September 2018). Google Scholar

18. Debiolles A, Oukhellou L, Aknin P, et al. Track circuit automatic diagnosis based on a local electrical modelling. In: 7th World Congress on Railway Research (WCRR 2006), Montreal, Canada, 2006. Google Scholar

19. KVH Industries, Inc. GEO FOG 3D INS, 2016. Brochure, www.kvh.com/Commercial-and-OEM/Gyros-andInertial-Systems-and-Compasses/Gyros-and-IMUs-andINS/INS/GEO-FOG-3D.aspx (accessed 15 September 2018). Google Scholar

20. Bombardier Inc . EBI track 200 TI21 audio frequency track circuit technical manual, Montreal: Bombardier Inc, 2011. Google Scholar

21. Allen, JB, Rabiner, LR. A unified approach to short-time Fourier analysis and synthesis. Proc IEEE 1977; 65: 1558-1564. Google Scholar, ISI

22. OpenStreetMap (openstreetmap.org) and contributors, CC-BY-SA (creativecommons.org) (accessed 15 September 2018). Google Scholar 